de, oder eines moderat erhöhten CRP(C-reaktives Protein)-Werts $\geq 0,32$ $(\mathrm{p}=0,186)$ gesehen. Signifikant häufiger lag eine Harnwegsinfektion vor, wenn der CRP $\geq 1,50 \mathrm{mg} / \mathrm{dl}$ betrug oder eine auffällige Urinanalyse vorlag $(\mathrm{p}=$
0,001). Eine manifeste Harnwegsinfektion sei bei Patienten mit akuter Nierenkolik mit einer Inzidenz von 13,3 \% eher selten, schlossen die Autoren. Eine Antibiotikaprophylaxe sei daher nicht notwendigerweise $\mathrm{zu}$ verabreichen, auch dann nicht, wenn die Leukozytenzahl erhöht ist. Bei einer CRP-Erhöhung $<1,5 \mathrm{mg} / \mathrm{dl}$ und ohne auffällige Urinproben könne der Antibiotikagebrauch ebenfalls eingeschränkt werden.

Dr. Ine Schmale

\title{
Änderungen im Lebensstil können Menschen mit Nykturie helfen
}

\begin{abstract}
$\mathrm{D}$ ie Nykturie schränkt bei älteren Menschen häufig das Schlafvermögen und damit die Lebensqualität ein. Dabei ist es offensichtlich möglich, über die Änderung der Ernährung und die Behandlung von anderen schlafbeeinträchtigenden Störungen das Leiden zu vermindern. Ein womöglich leicht zu ändernder Lebensstil bei älteren Menschen mit Nykturie ist die Reduktion der Salzaufnahme, so das Ergebnis einer japanischen Studie von Matsuo Tomohiro, Nagasaki/Japan, und Kollegen [Tomohiro M, et al. EAU 2017, Poster \#399]. In Japan ist die Salzaufnahme durch die traditionelle Ernährung generell sehr hoch. Daher wurden 321 Japaner und Japanerinnen, die von einer hohen Salzaufnahme über die Nahrung berichteten und unter Nykturie litten, über eine salzarme Ernährung unterrichtet und zu Änderungen der Häufigkeit nächtlicher Toilettengänge infolge der verrin-
\end{abstract}

gerten Salzzufuhr befragt. Der Erfolg der Aufklärung wurde über einen Zeitraum von zwölf Wochen biochemisch gemessen. 223 der Studienteilnehmer schafften es, die Salzaufnahme von täglich $10,7 \mathrm{~g}$ auf $8,0 \mathrm{~g}$ zu reduzieren und erreichten damit die durchschnittliche nächtliche Frequenz von 2,3 Toilettengängen auf 1,4 zu senken. Bei 98 Studienteilnehmern, die ihre tägliche Salzaufnahme von 9,6 g auf 11,0 g erhöhten, wurde im Gegensatz dazu eine Steigerung der Frequenz von 2,3 auf 2,7 Toilettengänge pro Nacht beobachtet. Wurde die Salzaufnahme reduziert, musste die Toilette auch tagsüber weniger häufig aufgesucht werden. Die Beantwortung eines Standardfragebogens zur Lebensqualität („,core lower urinary tract symptom score-quality of life", CLSSQoL) wies auf eine deutliche Verbesserung derselben durch die Reduzierung der nächtlichen Toilettengänge hin.
Auch die Behandlung einer obstruktiven Schlafapnoe (OSA) kann zur Reduktion von nächtlichen Toilettenepisoden führen, fanden niederländische Forscher um Sajjad Rahnama'i, Maastricht/Niederlande, heraus [Degalliers S, et al. EAU 2017, Poster \#566]. 256 Patienten, von denen 206 männlich sowie 50 weiblich waren, denen aufgrund der Schlafapnoe eine CPAP(„continuous positive airway pressure")-Maske verordnet wurde, nahmen an der Studie teil. $69 \%$ dieser Patienten berichteten von mehr als einem Toilettengang pro Nacht. Mit dem Gebrauch der Maske wurde bei $65 \%$ der Patienten eine Reduktion des nächtlichen Harndrangs beobachtet. Es sei vielleicht überraschend, dass Atemprobleme eine exzessive Urinproduktion anregen, aber das Problem sei durchaus real, kommentierte Marcus Drake, University of Bristol, für die EAU. Daher sei der Hinweis auf diesen Zusammenhang und der mögliche Nutzen der OSA-Therapie sehr hilfreich für die Praxis.

Dr. Ine Schmale

\section{Erektile Dysfunktion: erste Erfolge mit Stammzelltransplantation}

$D$ ie Stammzelltransplantation könnte künftig eine neue Therapieoption für Männer mit erektiler Dysfunktion (ED) darstellen, bei denen die Kontinenz erhalten ist. Darauf weist erstmals eine Phase-I-Studie hin [Haahr MK, et al. EAU 2017, Poster \#368]. In den letzten Jahren hatten mehrere Arbeitsgruppen an der Entwicklung von Stammzellen zur Behandlung der ED gearbeitet, doch jetzt ist es erstmals gelungen, dass betroffene Männer nach dieser Therapie wieder spontan Geschlechtsverkehr haben konnten. An der einarmigen, mo- nozentrischen Studie nahmen 21 Patienten mit ED nach radikaler Prostatektomie teil, bei denen konventionelle EDBehandlungen inklusive der Anwendung von Phosphodiesterase-5-(PDE -5)-Hemmern und Prostaglandin-E1(PGE1)-Analoga keinen Erfolg zeigten. Die Autoren isolierten autologe regenerative Stammzellen aus dem Fettgewebe (,autologous adipose derived regenerative stem cells", ADRC) der Teilnehmer unter Vollnarkose mittels Liposuktion und injizierten die Zellen unmittelbar anschließend in deren Corpus caverno- sum. Untersuchungen erfolgten ein, drei, sechs und zwölf Monate nach der ADRC-Transplantation. Hierbei wurden die erektile Funktion anhand des Fragebogens IIEF-5 (,international index of erectile function") sowie alle $\mathrm{Ne}$ benwirkungen erfasst. Schwere Nebenwirkungen traten nicht auf, lediglich acht kleinere Vorfälle in Zusammenhang mit der Liposuktion wurden berichtet, unter anderem Hämatome und empfindliche Haut im Abdominalbereich. Insgesamt ließ sich die Erektionsfunktion bei acht von 15 (53 \%) Männern wieder herstellen, die vor dem Eingriff kontinent gewesen waren. Sie konnten nach sechs Monaten wieder Geschlechtsverkehr ausüben, dieser Ef- 